\title{
Simulation of LIDAR-based aircraft wake vortex detection using a bi-Gaussian spectral model
}

\author{
Sébastien Lugan, Laurent Bricteux, \\ Benoît Macq, Piotr Sobieski \\ and Grégoire Winckelmans \\ Université catholique de Louvain \\ Faculté des Sciences Appliquées \\ 1348 Louvain-la-Neuve, Belgium
}

\author{
Damien Douxchamps \\ Nara Institute of Science and Technology \\ Image Processing Laboratory \\ 630-0192 Japan
}

\begin{abstract}
A new spectral model of the return signal from a LIDAR Doppler wake vortex detector is proposed. It has been experimentally discovered during ground-based and flight test campaigns but suffered a lack of theoretical evidence. Using high resolution fluid simulations of wake vortices, we highlight the physical meaning of this model. Comparisons with the traditional single Gaussian model show the superiority of this new approach is consistent with previous experimental results.
\end{abstract}

\section{INTRODUCTION}

Wake vortices are large rolling air masses generated by aircraft as a result of their lift. They present a significant danger during the landing and take-off phases and limit the throughput of airports[1]. They can be detected using an infrared Doppler LIDAR, using suspended atmospheric dust as tracers. Two main configurations are possible for detection: in the transverse plane (perpendicular to the aircraft trajectory) or axially (the LIDAR axis is parallel to the aircraft trajectory). The first one is easier because the air motion is mainly transverse in a vortex and thus the Doppler LIDAR sensor can see large particle velocity differences [2] [3]; however it only provides information about the vortex in a transverse plane and not along its whole spatial extension. Axial detection is more difficult because axial wind velocities are much lower, however this approach is necessary to obtain a more complete information on the vortex. Such a scanning configuration is presented on Fig. 1. In fact, the axial wind velocities are very low, so that the axial detection will be based more on turbulence than on speed measurements. The return spectrum from the Doppler LIDAR is analysed to obtain those information. Traditionally this has been done by considering either first and second order statistical moments of the spectrum of the return signal [4][5], which is similar to the classic approach of modelling the spectrum with a single Gaussian curve. However, if such a model succeeds very well when considering a turbulent atmosphere without vortex, Douxchamps [6] noted that it quickly leads to an important residue (thus meaning an imperfect fitting of the spectrum) when considering vortex activity regions. This prompted the introduction of a biGaussian model. In this paper we will present wake vortex simulations that confirm these experimental findings.

\section{FLUID DYNAMICS SIMULATION}

\section{A. Simulation technique and flow description}

The numerical simulation performed here aims to simulate the passage of heavy aircraft in a turbulent atmosphere. This computation was carried out in two steps. First, we computed the evolution of a turbulent atmosphere down to a prescribed level of dissipation: this sets the strength of the background turbulence which is chosen here as medium. Then, we insert a pair of Hallock-Burnham (low order algebraic) vortices to simulate the rolled up vortex system after passage of the aircraft. The initial total circulation of each vortex, $\Gamma_{0}$, is an image of the intensity of the vortex. $\Gamma_{0}$ can be estimated by equating the lift and the weight of the aircraft: $M g=\rho U b_{0} \Gamma_{0}$, where $M$ is the mass of the aircraft, $U$ its velocity, $\rho$ the air density, and $b_{0}$ the spacing between the wake vortex centroids (usually, one can estimate $b_{0}=\frac{\pi}{4} b, b$ being the wingspan). The initial descent velocity of the vortex wake, due to the mutual vortexinduced velocities, is:

$$
V_{0}=\frac{\Gamma_{0}}{2 \pi b_{0}} .
$$

This velocity is also used to define a dimensionless time $t^{*}=t \frac{V_{0}}{b_{0}}$.

The physical parameters of the simulated wake vortex flow are given in the table I.

TABLE I

DIMENSIONAL PARAMETERS OF THE WAKE VORTEX FLOW AND OF THE ATMOSPHERIC TURBULENCE.

\begin{tabular}{|c|c|c|}
\hline Vortex circulation & $\Gamma_{0}$ & $400 \mathrm{~m}^{2} / \mathrm{s}$ \\
\hline Vortex spacing & $b_{0}$ & $50 \mathrm{~m}$ \\
\hline Dissipation level & $\epsilon$ & $10^{-4} \mathrm{~m}^{2} / \mathrm{s}^{3}$ \\
\hline Periodicity length & $L_{x}, L_{y}, L_{z}$ & $4 b_{0}(200 \mathrm{~m})$ \\
\hline
\end{tabular}

The Navier-Stokes equations are here solved using a hyperviscosity subgrid scale model. This allows simulating fluid flows at very high Reynolds number (essentially infinite). The equations being solved are thus:

$$
\frac{\partial \mathbf{u}}{\partial t}+(\mathbf{u} \cdot \nabla) \mathbf{u}=-\nabla P+(-1)^{p+1} \nu_{h} \nabla^{2 p} \mathbf{u}
$$




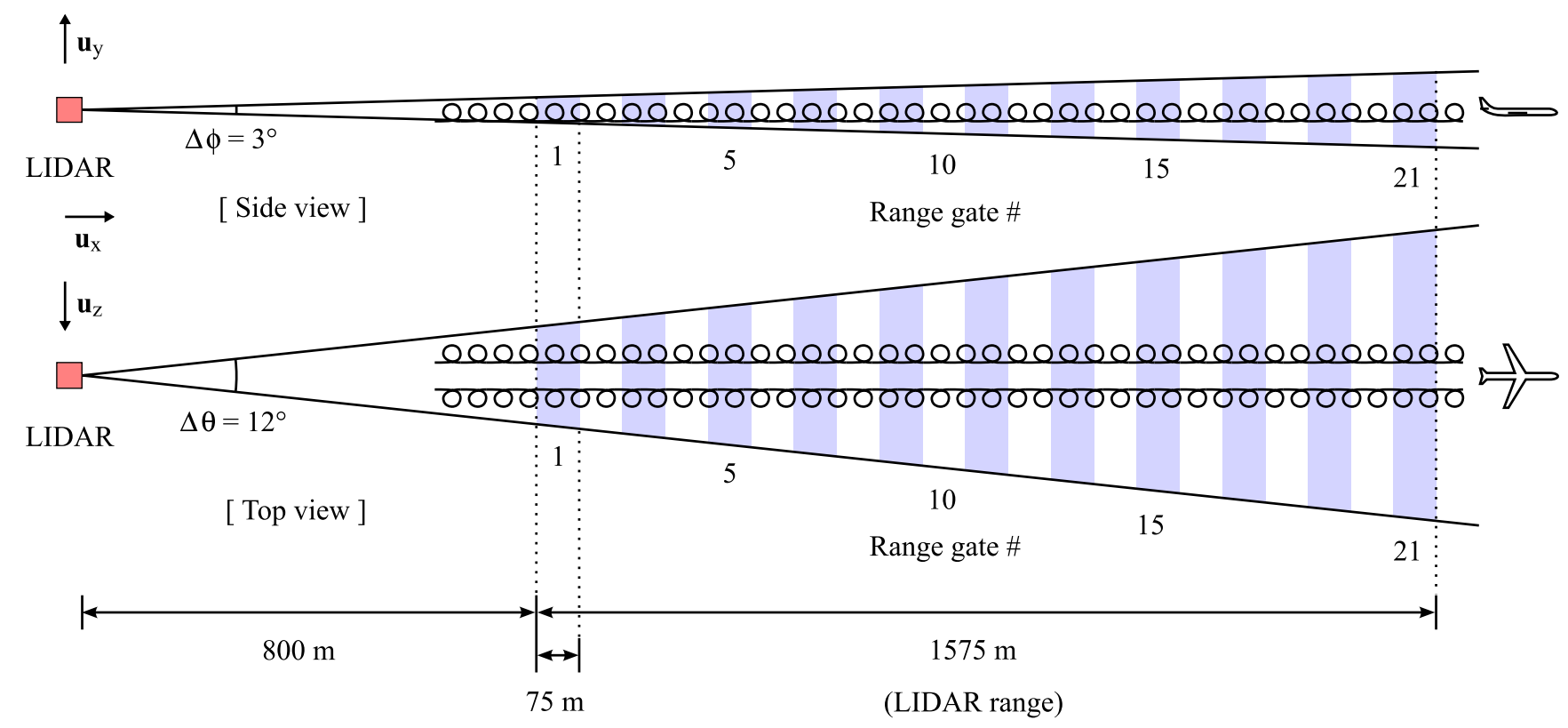

Fig. 1. Configuration of the virtual LIDAR scanning; the circles denote the location of the wake vortices.

together with the incompressibility constraint $\nabla \cdot \mathbf{u}=0$ $(P=p / \rho$ is the reduced pressure). In this study, we took $p=8$. The numerical solution of the incompressible NavierStokes equations are computed using a standard FourierGalerkin projection in all three Cartesian directions. This implies that the boundary conditions must be periodic in the three directions of space. The time integration of the equations is carried out using a third order Runge-Kutta scheme. We use a computational box of $256^{3}$ grid points, ensuring that the core of the vortices are well captured. The size of the computational box is chosen to minimize the periodicity effects.

\section{B. Results of the wake vortex simulation}

The value of the axial vorticity $\omega_{x}$ displayed in Fig. 2 is chosen to visualize the flow topology. One can see the vortex tubes and the surrounding vorticity due to the background turbulence and the turbulence generated by the vortex itself.

The axial velocity signature is of particular importance in the framework of airborne LIDAR detection. In this case, the lines of sight (LOS) of the LIDAR are oriented almost parallel to the vortices axis. This implies that the velocity component which has the most important contribution to the Doppler shift is the $u_{x}$ component which is aligned with the wake vortices. One can think that a vortical flow such as the one found in an aircraft wake vortex system is purely tangential (the flow has only $u_{y}$ and $u_{z}$ component) but, in our case, where turbulence is present, an axial $u_{x}$ velocity component is present. One can see on Fig. 3 that the values of this axial velocity component $\left(u_{x} \approx 1 \mathrm{~m} / \mathrm{s}\right)$ are non-negligible compared to the maximal tangential velocity $\left(u_{\max } \approx 12 \mathrm{~m} / \mathrm{s}\right)$.

\section{GeOMETRIC LIDAR SIMULATIONS}

In order to assess the validity of the bi-Gaussian model, we used the high-resolution fluid simulation $\left(256^{3}\right.$ LES) of a wake

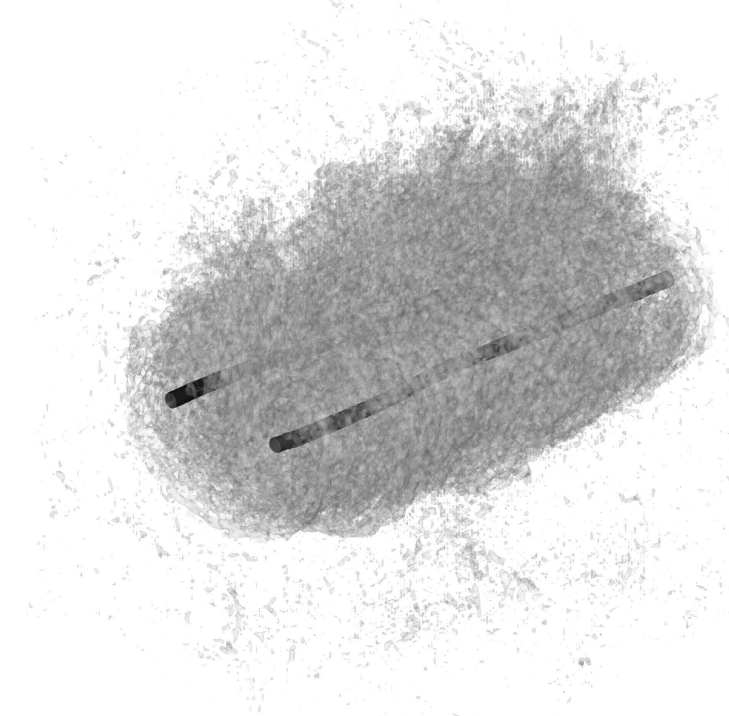

Fig. 2. Visualization of the flow field using isosurfaces of vorticity norm at time $t^{*}=2$.

vortex pair taken at various ages (Fig. 3), and we appended on all sides the turbulence field without the vortices (Fig. 4).

An arbitrary position of the virtual LIDAR sensor was chosen (as illustrated on Fig. 1), corresponding to the worse in-flight scenario of on-board wake vortex detection: a purely axial view of the vortices, where the tangential particles velocities have minimum influence.

Lines of sight, originating from the virtual sensor and going into the scanned volume were then considered, within a range defined by $\theta \in\left[-6^{\circ}, 6^{\circ}\right]$ and $\phi \in\left[-1.5^{\circ}, 1.5^{\circ}\right], \theta$ and $\phi$ corresponding respectively to the horizontal (azimuth) and vertical (elevation) deviation angles of the sensor's scanner. 


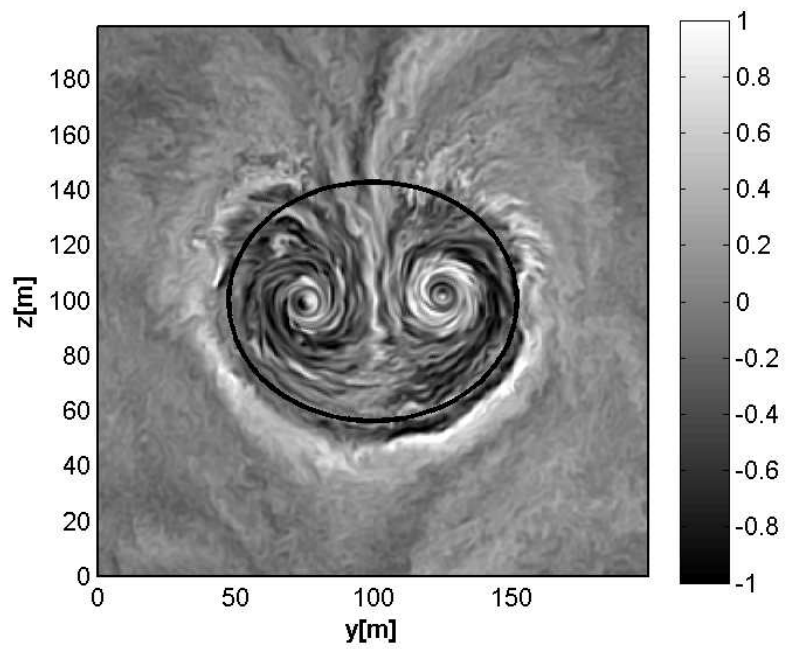

Fig. 3. Visualization of the axial velocity component $u_{x}$ at time $t^{*}=2$ for the $256^{3}$ Large Eddy Simulation (LES). The thick line shows the theoretical Rankine oval.

This setup considers the same scanning ranges as those used during M-FLAME ground tests [4] and I-WAKE flight-tests [6].

Assuming a virtual acquisition frequency of $500 \mathrm{MHz}$, we collected the corresponding radial velocities along the LOS, as an estimator of the Doppler shift (which is proportional to the velocity of the particles) that would have been detected by a real LIDAR sensor. However, no LIDAR nor optical simulation has been performed here, since velocity data were directly exploited. We were thus assuming an ideal radial velocity sensor. The histogram of this velocity vector was then computed from the individual velocity values along the LOS. It would correspond to the discrete Fourier Transform (DFT) of the temporal LIDAR return signal in the case of a real LIDAR sensor. In order to gather a statistically significant amount of data, we aggregated the LOS within a large region $\left(1.2^{\circ} \times 1.2^{\circ}\right)$.

\section{BI-GAUSSIAN MODEL}

The traditional, single-Gaussian model is:

$$
f_{1}(x)=\frac{1}{\sigma \sqrt{2 \pi}} \exp \left(-\frac{(x-\mu)^{2}}{2 \sigma^{2}}\right)
$$

where $\sigma^{2}$ represents the variance and $\mu$ the mean value of the Gaussian distribution.

More accurate results can be achieved if we consider that the observed LIDAR return signal spectrum is the juxtaposition of the spectrum generated by 1) a population of particles affected by the general turbulent behavior of the atmosphere only and 2) a population of particles affected by the vortical activity. We may consider that each of these populations has a distinct Gaussian probability density function and their juxtaposition could thus be modeled by the sum of two Gaussian distributions (as suggested in [6]). The first one, associated to the global turbulence, has the same mean and variance as those found when fitting the general turbulence (outside any vortex) with a single Gaussian model. The second one, associated to the local vorticity, is much broader and thus of larger variance.

The high-resolution fluid simulation shows that, even when considering an axial scanning of the vortex tubes, the velocity dispersion of the particles is much larger inside the vortices than in the general turbulence outside the vortices.

The double Gaussian model is then:

$$
\begin{aligned}
f_{2}(x) & =\frac{1}{\sigma_{t} \sqrt{2 \pi}} \exp \left(-\frac{\left(x-\mu_{t}\right)^{2}}{2 \sigma_{t}^{2}}\right) \\
& +\alpha_{v} \frac{1}{\sigma_{v} \sqrt{2 \pi}} \exp \left(-\frac{\left(x-\mu_{v}\right)^{2}}{2 \sigma_{v}^{2}}\right)
\end{aligned}
$$

where $\sigma_{t}^{2}$ represents the variance and $\mu_{t}$ the mean value of the Gaussian distribution caused by the general turbulence, $\sigma_{v}^{2}$ the variance, $\mu_{v}$ the mean and $\alpha_{v}$ the relative contribution of the second Gaussian distribution, caused by the vortex.

\section{Results}

Simulations have been performed in an axial case, meaning that the LOS are almost perfectly aligned with the center of the vortex axes. Note that, even in this case, for geometrical reasons, the LOS reaching the vortices will not be perfectly aligned with each of the vortices' axis. The effect of this nonperfect alignement, however small, will be that some tangential component of the particles velocities will be detected by the Doppler LIDAR.

Results from the simulation were used to create a histogram of wind speeds which was then fitted using a LevenbergMarquardt non-linear regression with a single and bi-Gaussian model.

In order to illustrate the fundamental difference between the histograms within and without the wake vortices, we will consider two regions of interest: region 1, located outside any vortex, and region 2, located inside the right vortex, both located within the scanned volume (Fig. 4). The corresponding histograms are plotted respectively on Fig. 5 and Fig. 6.

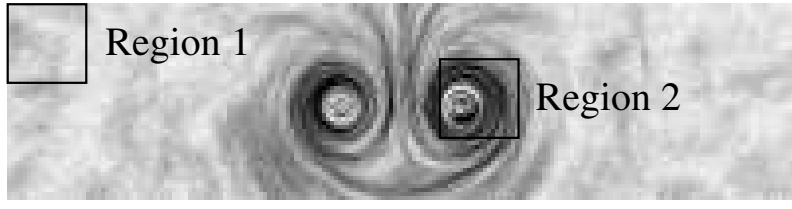

Fig. 4. Location of the two regions of interest within the scanned area. The resolution shown is that of the LIDAR shot simulation: here $200 \times 50$.

In the first case, outside the vortex (Fig. 5), the distribution of particle velocity is expectedly Gaussian and consequently can be fitted with good accuracy by a single Gaussian model. Inside the vortex (Fig. 6), however, a single Gaussian fit departs significantly from the observed distribution. The situation would be even worse if the single Gaussian fit would only rely on the upper part of the curve (e.g. to avoid biases due to background noise level). Using the bi-Gaussian model on the same distribution we can observe a much better fit of the particle velocity distribution as observed in [6]. In fact, the second (broadest) Gaussian distribution ("vortex contrib." on Fig. 6) directly characterizes the vorticity and leads to a much more accurate detection of wake vortices. 


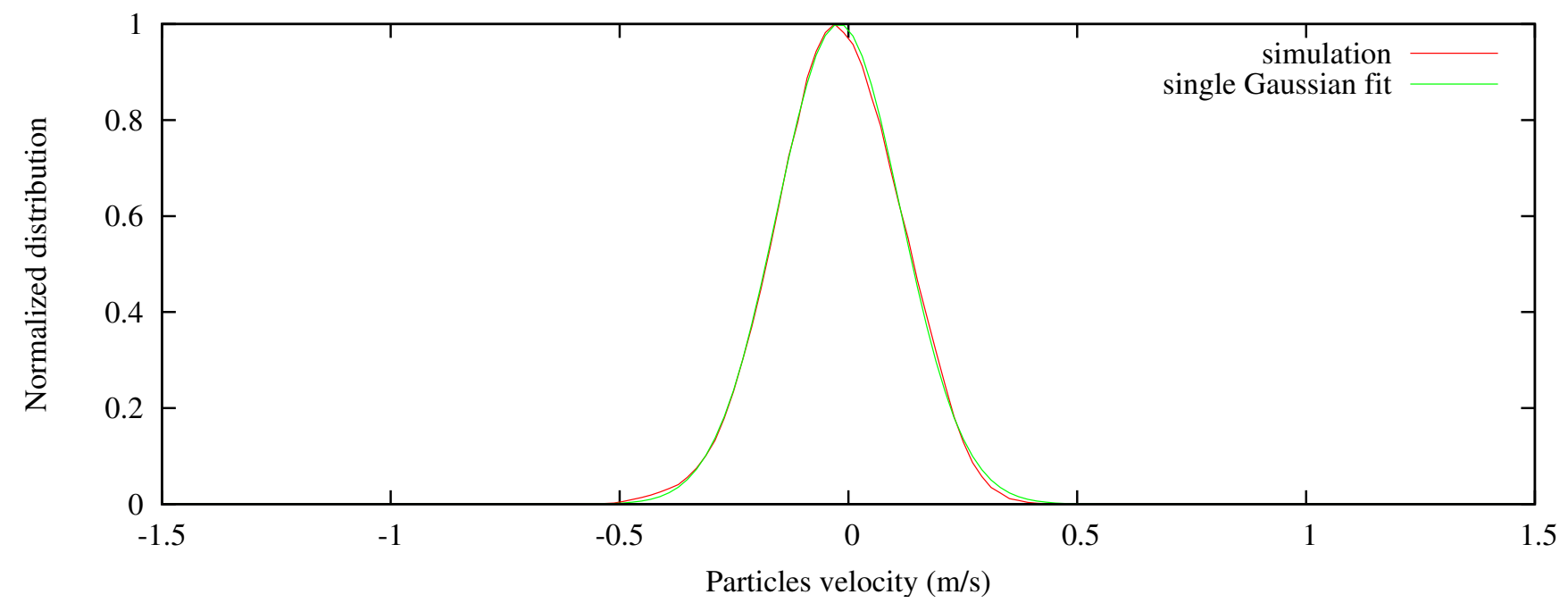

Fig. 5. Normalized distribution of the velocities within region 1 (outside any wake vortex, but still inside turbulence); parameters of the fitted Gaussian: $\mu=-0.020 \mathrm{~m} \cdot \mathrm{s}^{-1} ; \sigma=0.136 \mathrm{~m} \cdot \mathrm{s}^{-1}$.

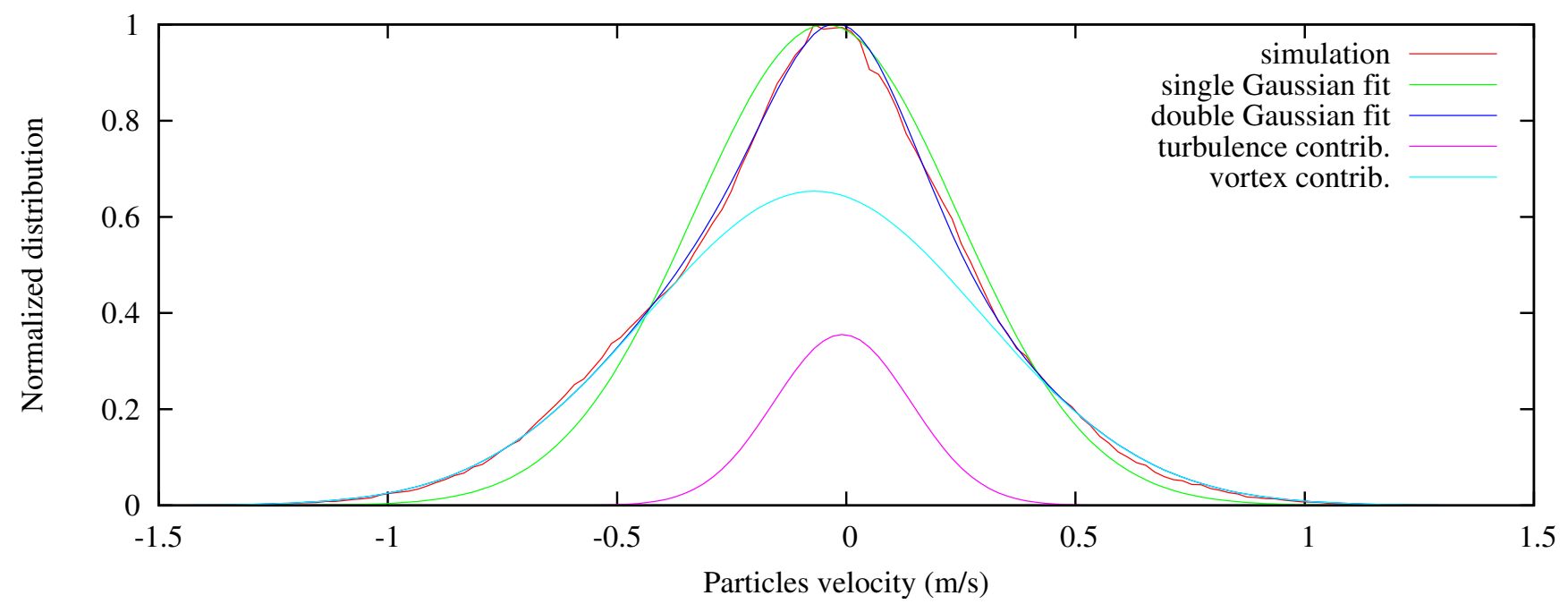

Fig. 6. Normalized distribution of the velocities within region 2 (inside the right vortex); parameters of single Gaussian fit: $\mu=-0.045 \mathrm{~m} \cdot \mathrm{s}-1 ; \sigma=$ $0.288 \mathrm{~m} \cdot \mathrm{s}^{-1}$; parameters of the double Gaussian fit: $\mu_{t}=-0.009 \mathrm{~m} \cdot \mathrm{s}^{-1} ; \sigma_{t}=0.149 \mathrm{~m} \cdot \mathrm{s}^{-1} ; \alpha_{v}=1.839 ; \mu_{v}=-0.069 \mathrm{~m} \cdot \mathrm{s}^{-1} ; \sigma_{v}=0.365 \mathrm{~m} \cdot \mathrm{s}^{-1}$.

\section{CONClusions}

Our simulations showed that the histogram of particles velocities within a wake vortex that is axially scanned by a LIDAR can be accurately modeled by a bi-Gaussian distribution, when several neighbour LOS are aggregated. As a consequence, the classical single Gaussian model is not optimal in order to detect wake vortices in the axial or nearly axial cases. The correlation between experimental measurements and our simulations are good; this is a significant step towards an axial wake vortex detection system and, ultimately, towards more safety and capacity for airports.

\section{ACKNOWLEDGMENTS}

This work was supported by the Commission of the European Union (Fidelio project) and a grant from the DGTRE Région Wallonne (LASEF project).

\section{REFERENCES}

[1] J. N. Hallock, C. Tung, and S. Sampath, "Capacity and wake vortices," in Proc. Intl. Congress of Aerospace Sciences (ICAS'02), Toronto, Canada, Sept. 2002.

[2] J. M. Vaughan, K. O. Steinvall, Christian Werner, and Pierre-Henri Flamant, "Coherent laser radar in Europe," Proc. IEEE, vol. 84, no. 2, pp. 205-226, Feb. 1996.

[3] B. Lamiscarre, B. Christophe, C. Fournet, J. Lemorton, L. Poutier, and A. Oyzel, "Nouveaux capteurs pour l'amélioration de la sécurité et de l'efficacité des mouvements en zone aéroportuaire," in Proc. Sème Journée AAAF, Toulouse, France, Jan. 1999.

[4] M. Keane, D. Buckton, M. Redfern, Christoph Bollig, Carsten Wedekind, and Friedrich Kopp, "Axial detection of aircraft wake vortices using doppler lidar," Journal of Aircraft, vol. 39, no. 5, pp. 850-861, Sept. 2002.

[5] M. J. Levine, "Power spectrum parameter estimation," IEEE Trans. Inform. Theory, vol. 11, pp. 100-107, 1965.

[6] D. Douxchamps, Multidimensional Photogrammetry of Short-Lived Events, Thèse de doctorat, Université catholique de Louvain, Louvainla-Neuve, Belgium, Oct. 2004. 